\title{
Pulmonary metastasectomy for colorectal cancer: Making the case for a randomized controlled trial in the zone of uncertainty
}

\author{
Francesca Fiorentino, $\mathrm{PhD},{ }^{\mathrm{a}}$ and Tom Treasure, MD, MS, FRCS, FRCP
}

Survival after pulmonary metastasectomy, from the earliest to the most recent reports, has been related to the number of metastases and the time to their appearance. The fewer the metastases and the longer the interval between primary surgery and metastasectomy, the better is survival. ${ }^{1}$ Many clinical reports (by now there are $>100$ for colorectal cancer) have implied that survival is gained by pulmonary metastasectomy and have encouraged widening the indications. A survey of members of the European Society of Thoracic Surgeons, from November 2006 through January 2007, found that the large majority of responding surgeons $(86 \%)$ placed no upper limit on the number of pulmonary metastases they were prepared to resect and 64\% would perform a metastasectomy within a year of the primary cancer surgery. ${ }^{2}$ An alternative explanation for the association between metastasectomy and longer survival is that careful selection of patients with favorable prognostic features, which is central to the management of these patients, gathers in patients likely to survive longer. Observational studies with larger numbers of patients and better statistical analyses have shown that the old rules still apply and a more liberal implementation of pulmonary metastasectomy is associated with diminishing returns or worse, and it becomes evident that benefit in these patients is improbable and therefore they should be excluded. ${ }^{1,3}$ In this brief review we examine observational data and mathematic modeling. We conclude that a clinical trial, focusing on the zone of uncertainty, is needed. The Pulmonary Metastasectomy in Colorectal Cancer (PulMiCC) trial, based on that reasoning, is recruiting patients in Europe (http://www. rbht.nhs.uk/research/cteu/projects/respiratory-disease/ pulmicc/).

\footnotetext{
From the Department of Cardiothoracic Surgery, ${ }^{\mathrm{a}}$ National Heart and Lung Institute, Imperial College London; Clinical Operational Research Unit, ${ }^{\mathrm{b}}$ University College London, London, United Kingdom.

Funded in part by the British Heart Foundation (to Dr Fiorentino).

Disclosures: The authors have nothing to disclose with regard to commercial support. Received for publication March 13, 2013; revisions received June 15, 2013; accepted for publication June 18, 2013; available ahead of print Aug 5, 2013.

Address for reprints: Tom Treasure, MD, MS, FRCS, FRCP, Clinical Operational Research Unit, University College London, 4 Taviton St, London WC1H 0BT, United Kingdom (E-mail: tom.treasure@gmail.com).

J Thorac Cardiovasc Surg 2013;146:748-52

$0022-5223 / \$ 36.00$

Copyright (c) 2013 by The American Association for Thoracic Surgery

http://dx.doi.org/10.1016/j.jtcvs.2013.06.025
}

\section{THE LIMITS TO PULMONARY METASTASECTOMY FROM THE ANALYSIS OF OBSERVATIONAL DATA}

The number of metastases has a large effect on survival after pulmonary metastasectomy, which has been observed in individual reports and in aggregated data since the International Registry of Lung Metastases. ${ }^{4}$ Solitary metastases constitute $60 \%$ of the 3504 cases in the most inclusive of the systematic reviews. ${ }^{5}$ The favorable effect of a longer interval after primary resection is also a consistent observation and the average interval in published series is 2 to 3 years. ${ }^{1}$ To extrapolate from series dominated by patients with inherently favorable prognostic features, to a wider group with unfavorable characteristics, will not be associated with as favorable survival rates.

A combined report from Duke and Memorial SloanKettering Cancer Center in 2009 appeared to be the first to recommend explicit limits to metastasectomy for colorectal cancer. ${ }^{3}$ The investigators reported that of 44 patients with 3 or more lesions who had a pulmonary metastasectomy less than 1 year after the primary resection, "none was cured by operation."3 Their recommendation is quite explicit that medical management alone should be considered standard for patients who have both 3 or more pulmonary metastases and less than a 1-year interval since the primary cancer resection. In February 2013 the latest systematic review, including a sophisticated meta-analysis of surgical follow-up studies, reported that patients with more than a solitary metastasis were significantly more likely to have a recurrence. ${ }^{1}$ This was a consistent finding among studies from 2001 to 2011, from which they derived the data for analysis providing "robust information regarding nearly 3000 patients," but then opined, "it seems currently unfair to deny surgery for those patients with two to four lesions." 1 If the evidence indicates that the likelihood of benefit is very uncertain then it seems to us to be fair to communicate that explicitly to patients so that they fully appreciate that avoiding unavailing surgery is a reasonable course of action. Furthermore, the responsibility should be borne by the medical profession to obtain the evidence to better inform patients' choice.

\section{MATHEMATIC MODELING OF SURVIVAL OF PATIENTS WITH METASTATIC COLORECTAL CANCER}

Before considering a trial it is important to explore all available means of discerning the signal, which in this instance is longer survival attributable to metastasectomy, 
from the noise, which is represented by heterogeneity of pathology and the effects of multiple treatments. Two large reports from the 1990 s with 144 and 159 patients ${ }^{6,7}$ provided data on 2 prognostic features that also are recorded for patients in the Thames Cancer Registry. ${ }^{8}$ One is the colorectal cancer stage at the time of primary resection, and the other is the so-called disease-free interval. A modeling exercise has been performed using data from the registry and from the published cohorts. Registry patients with a similar mix of cancer stage were identified. Among these were selected patients who had survived for a "death-free interval" 6 (and thus were available for further surgery) similar to the disease-free interval of patients reported in the cohorts. The survival of patients included in the models, selected on those 2 criteria, was similar to that in the reported metastasectomy cohort (Figure 1). It is notable that patients with metastases at the time of registration had a survival rate of more than $5 \%$ at 5 years. The process of clinical selection for metastasectomy preferentially would include these survivors. ${ }^{9}$ The models indicate uncertainty concerning benefit attributable to pulmonary metastasectomy but cannot prove that this surgery has no effect; a randomized trial is required.

\section{PREDICTIVE OR PROGNOSTIC?}

It is important to emphasize the distinction between the words prognostic and predictive. ${ }^{10} \mathrm{~A}$ prognostic factor is a variable that can be known before starting the treatment. Variation in survival is influenced by these factors regardless of which treatment the patient receives. The number of metastases and the interval before they appear are prognostic factors, as is carcinoembryonic antigen (CEA). A predictive factor relates to treatment responsiveness. Thus, failure to achieve $\mathrm{R} 0$ resection is predictive of a poor outcome but cannot be known until after surgery and therefore cannot be used reliably in selection. Mediastinal nodal involvement is both an adverse prognostic feature when it is known before surgery and predictive of poorer survival if discovered at surgery. In reports of pulmonary metastasectomy, predictive and prognostic factors often are listed together, as they were by Gonzalez et al, ${ }^{1}$ who listed 4 factors that increased the probability of death by a factor of 2 in the meta-analysis: more than 1 metastasis, synchronous lung metastasis, involvement of thoracic lymph nodes, and increased prethoracotomy CEA.

\section{CEA AND SURGERY FOR ADVANCED COLORECTAL CANCER}

The CEA assay has an instructive history. Its use in surveillance after primary resection of colorectal cancer was evaluated in the Cancer Research Campaign CEA Second-Look Trial. This tested the concept that early recurrence in pelvic or mesenteric lymph nodes, the retroperitoneum, the liver, or at the colonic anastomosis
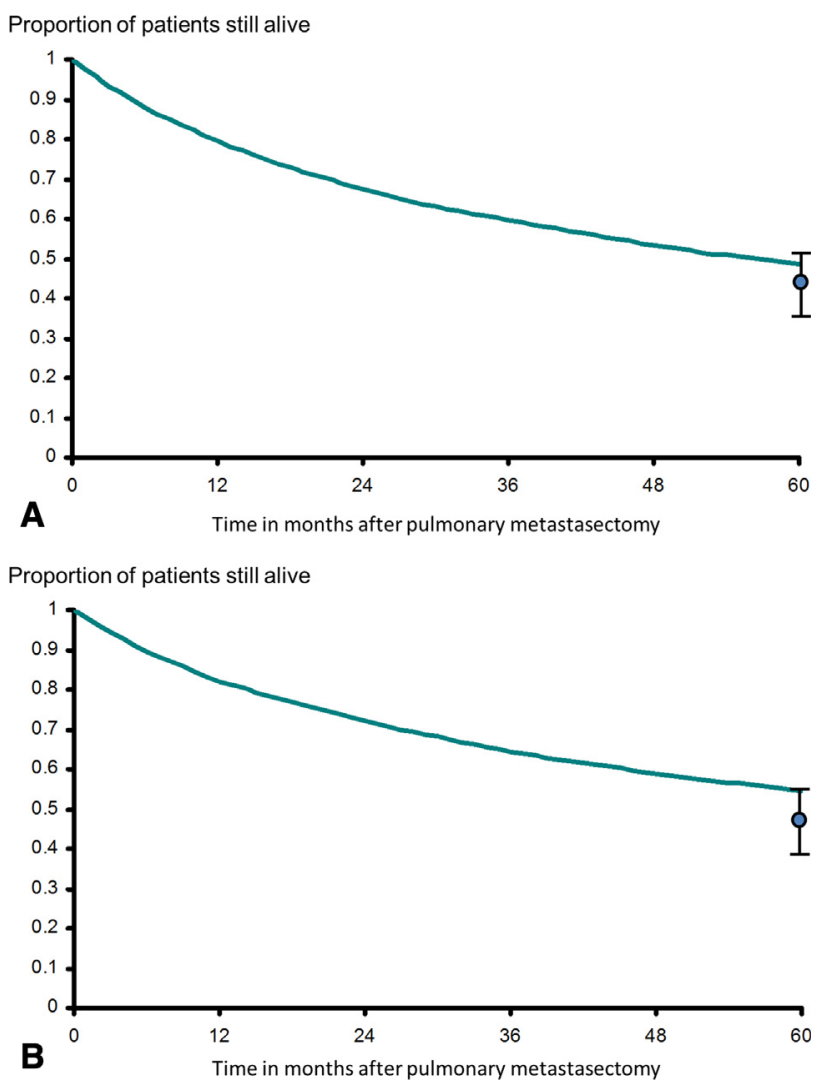

FIGURE 1. A, The data on the Dukes' stage mix and the disease-free interval in a cohort of 144 patients who had pulmonary metastasectomy were used to construct a model to estimate survival among patients in the Thames Cancer Register matched for Duke stage. The inclusion of registry patients in the model was conditional on having survived, to be available for metastasectomy, for an interval equivalent to the diseasefree interval provided in the clinical report of McCormack et al. ${ }^{6}$ The reported 5-year survival rate (with 95\% confidence intervals) is shown. $\mathrm{B}$, The same exercise performed with respect to a cohort of 159 patients in Japan. ${ }^{7}$ Reprinted with permission. ${ }^{8}$

might be resected successfully, providing a second chance of achieving cure. Patients with increased CEA were allocated randomly to have, or not to have, a second-look laparotomy. ${ }^{11}$ Patients undergoing a second-look laparotomy did not have better survival. The patients undergoing a second-look laparotomy had more investigations, hospitalizations, and surgery, but did not live longer. The trial was stopped by the data monitoring committee after 1447 patients had been registered and 216 patients had been randomized. Harm had been caused, without benefit, and it was improbable that any benefit would ever be shown.

Included in the second-look laparotomy protocol was mobilization of the liver in line with the growing practice of liver resection for colorectal metastases. ${ }^{12}$ There was, at the time, an opportunity to perform a trial of liver resection effectiveness. A power calculation published by the Mayo Clinic indicated that 36 randomized patients 
would have been sufficient to prove effectiveness of the metastasectomy if the 25\% 5-year survival rate among those undergoing liver resection was largely attributable to surgery. ${ }^{13}$ The trial was not performed and instead hepatic resection went into standard clinical care without secure evidence.

With respect to CEA we are now faced with a dilemma. When used in surveillance, after resection of primary colon cancer, an increase in CEA prompts further imaging. Lung nodules are the most visible recurrence, and are easily seen radiologically against the blackness of an air-filled lung. But a CEA increase has long been known to be associated with poorer survival after pulmonary metastasectomy. Here is the paradox: the same blood test that prompted referral for consideration of pulmonary metastasectomy may then be grounds to turn the patient away from thoracic surgery. CEA did not appear in the Duke-Memorial Sloan-Kettering Cancer Center study ${ }^{3}$ as a risk factor for death. This was probably because the range of CEA levels among surgical cohorts was restricted because clinicians applied the evidence already widely available. ${ }^{5}$

\section{THE CURRENT PRACTICE OF PULMONARY METASTASECTOMY FOR COLORECTAL CANCER}

For the Gonzalez et al ${ }^{1}$ meta-analysis, although the date limit on publications for inclusion was set at 2001, patients included were from as far back as the 1980s. How do these data for patient inclusion reflect current practice? There are newly published data from the Grupo Español de Cirugía Metástasis Pulmonares de Carcinoma Colo-Rectal (the Spanish Group for Surgery of Pulmonary Metastases from Colorectal Cancer). The study reported a prospective registry of 543 patients from 2008 to 2010 who represented an estimated $60 \%$ of all Spanish patients undergoing metastasectomy. ${ }^{14}$ In $55 \%$ of patients there was a solitary metastasis. The median interval between the primary cancer resection and metastasectomy was 28 months. This is consistent with the International Registry of Lung Metastases from the $1990 \mathrm{~s},{ }^{4}$ the collected series in the meta-analysis including patient from the $1980 \mathrm{~s},{ }^{1}$ and clinical reports back to the 1970 s. ${ }^{5}$ What is different is that in more recent practice metastatic disease was present in the liver in $29 \%$ of patients at one or more time points before pulmonary metastasectomy. ${ }^{14}$

Estimates of the degree of case selection are impossible to derive from the clinical reports of eminent institutions because we have no way of knowing the denominator from which they were drawn and many patients are secondary or tertiary referrals. The Spanish Group's study estimated that these 543 patients were at most $5 \%$ of the patients with lung metastases from colorectal cancer or $2.5 \%$ of all colorectal cancer patients, and they found that these rates are broadly comparable with Japanese and
Italian data on case selection. ${ }^{14}$ At this level of selection of patients based on prognostic factors (number, interval, and CEA) a $40 \%$ five year survival rate could be attributed entirely to selection, with no need for any effect of metastasectomy to explain the outcome (Figure 2).

\section{SYMPTOMS AND QUALITY OF LIFE}

None of the reviews provide any information on symptomatic benefit. This is not surprising: pulmonary metastasectomy is performed on patients with good performance status. Symptoms are not a feature of pulmonary metastases being considered for resection and therefore would not be retrieved on chart review. In fact, the terminal phase of the natural history of colorectal cancer is little influenced by any symptomatic effects of pulmonary metastases and they very infrequently contribute to death. In PulMiCC, patient-reported outcomes, measures of quality of life, and specifically respiratory symptoms, will be recorded. Respiratory benefit is not anticipated whereas detrimental effects must be ascertained in the evaluation of overall effectiveness of pulmonary metastasectomy.

\section{THE PulMiCC TRIAL}

A few patients with a solitary metastasis might have the only residue of their cancer removed and indefinite

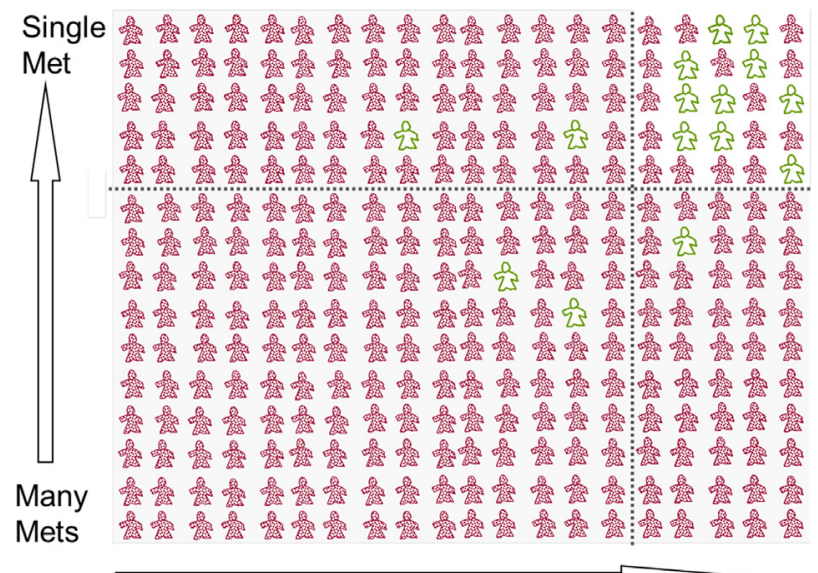

Increasing interval between the primary resection and the metastasectomy

FIGURE 2. Among 300 patients with pulmonary metastases, there are 15 patient $(5 \%)$ destined to live for 5 years after their primary colorectal cancer surgery based on cancer registry data that are on the conservative side. The patients have been sorted according to the number of metastases and the interval since the primary resection. The 15 patients destined to survive are likely to have fewer metastases and longer intervals and might be clustered as shown toward the top right of the matrix. If we now apply the usual selection criteria to select 1 in 12 patients, this might include 10 of the predestined survivors among these 25 patients. The $40 \%$ (10 of 25) inclusion of green figures is the result of sorting and selection, but incorrectly could be attributed to the fact that they underwent surgery. ${ }^{9}$ Reprinted with permission. ${ }^{9}$ 


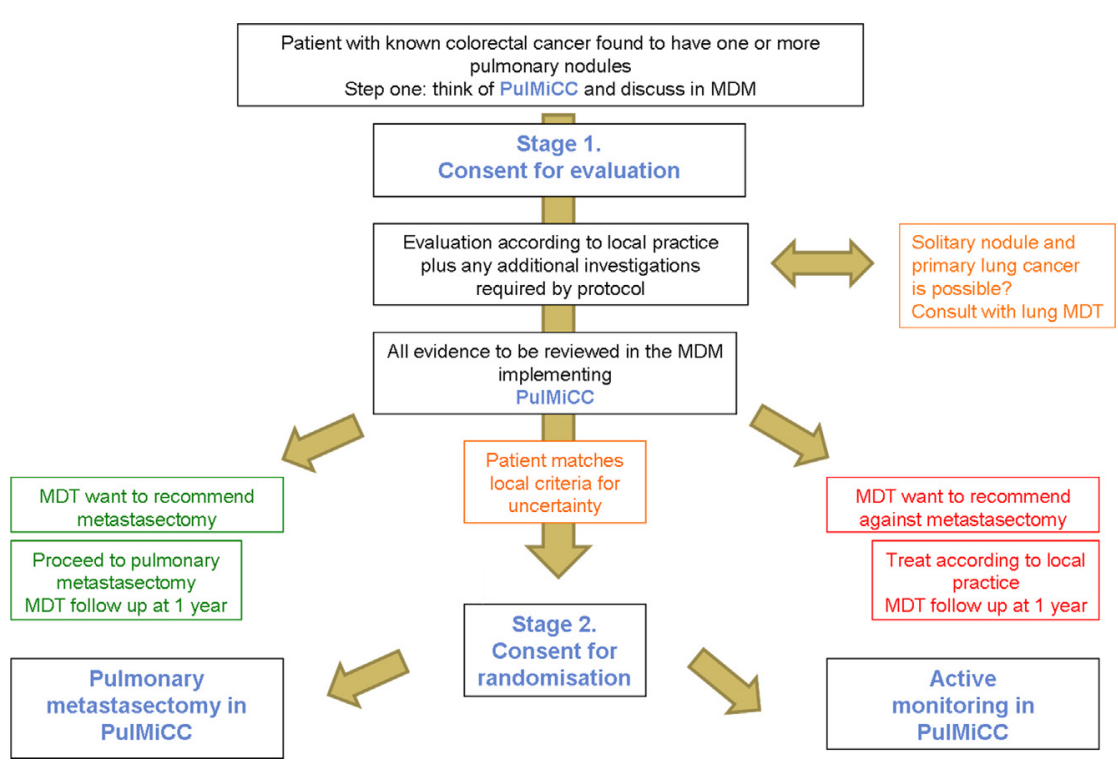

FIGURE 3. Trial flow chart. The decision-making area of the chart runs vertically in the center of the flowchart. Red areas of the chart indicate patients selected by the local multidisciplinary team to not have surgery, and green indicates those selected to have surgery. Amber indicates uncertainty. The first uncertainty (to the right) is whether a solitary nodule is a metastasis. The second (at the bottom) is whether there is clinical agreement on whether the patient should or should not have a metastasectomy. PulMiCC, Pulmonary Metastasectomy in Colorectal Cancer. MDT, Multidisciplinary team; $M D M$, multidisciplinary meeting.

survival attributed to the metastasectomy. How often that occurs cannot be derived from the available evidence so we concluded that a survival gain attributed to metastasectomy was unproven. The proposal therefore arose to put pulmonary metastasectomy to the fair test of a randomized controlled trial. Randomized controlled trials in surgery appear to some to be a forlorn hope: akin to putting a square peg in a round hole. ${ }^{15}$ However, there is a very substantial hole in our knowledge concerning the effectiveness of pulmonary metastasectomy; the trick surely is to use imagination and expertise in trial design to fashion a research peg to fit the hole in the evidence. The essence of the PulMiCC trial is that it is based on real-world practice: most patients with pulmonary metastases from colorectal cancer do not have a metastasectomy for a range of reasons related to the characteristics of the patient and the behavior of the cancer. Only a minority are selected. It follows that if there is yes for some and no for others, there must be debatable cases for which it seems unfair to deny ${ }^{1}$ surgery or for whom the benefit of the doubt is invoked. These are the patients in whom there is manifest uncertainty and to whom randomized allocation can be offered. The inclusion of patients thus is determined by local belief, practice, and convictions, and is not dictated by the clinical trial protocol. The trial design ensures balance within any given trial center. Variation in perception of where uncertainty lies broadens inclusion and will make the result more generalizable. Outcomes of interest in the study were survival and quality of life.
The trial therefore has 2 stages (Figure 3). In the first stage, without any commitment to randomization, patients who have had curative resection of their primary cancer and are found to have pulmonary metastatic disease, are fully assessed and fully informed, just as in current best practice. Many patients are found to have features that would make benefit improbable. At the other end of the distribution are those who have such favorable characteristics that the local team wants to recommend surgery and the patient wants to accept it. It is those in the middle, for whom uncertainty becomes manifest, who are offered random allocation. The PulMiCC trial is recruiting in Europe. Full details of the trial can be obtained from the trial center at Royal Brompton Hospital (http://www.rbht.nhs.uk/ research/cteu/projects/respiratory-disease/pulmicc/). More than 160 patients are in the study at the time of writing with approximately a third of them going forward for random allocation for whether or not to have pulmonary metastasectomy.

\section{References}

1. Gonzalez M, Poncet A, Combescure C, Robert J, Ris HB, Gervaz P. Risk factors for survival after lung metastasectomy in colorectal cancer patients: a systematic review and meta-analysis. Ann Surg Oncol. 2013;20:572-9.

2. Internullo E, Cassivi SD, Van Raemdonck D, Friedel G, Treasure T. Pulmonary metastasectomy: a survey of current practice amongst members of the European Society of Thoracic Surgeons. J Thorac Oncol. 2008;3:1257-66.

3. Onaitis MW, Petersen RP, Haney JC, Saltz L, Park B, Flores R, et al. Prognostic factors for recurrence after pulmonary resection of colorectal cancer metastases. Ann Thorac Surg. 2009;87:1684-8.

4. Pastorino U, Buyse M, Friedel G, Ginsberg RJ, Girard P, Goldstraw P, et al. Long-term results of lung metastasectomy: prognostic analyses based on 5206 cases. J Thorac Cardiovasc Surg. 1997;113:37-49. 
5. Fiorentino F, Hunt I, Teoh K, Treasure T, Utley M. Pulmonary metastasectomy in colorectal cancer: a systematic review and quantitative synthesis. $J R$ Soc Med. 2010;103:60-6.

6. McCormack PM, Burt ME, Bains MS, Martini N, Rusch VW, Ginsberg RJ. Lung resection for colorectal metastases. 10-year results. Arch Surg. 1992;127: 1403-6.

7. Okumura S, Kondo H, Tsuboi M, Nakayama H, Asamura H, Tsuchiya R, Naruke T. Pulmonary resection for metastatic colorectal cancer: experiences with 159 patients. J Thorac Cardiovasc Surg. 1996;112:867-74.

8. Utley M, Treasure T, Linklater K, Moller H. Better out than in? The resection of pulmonary metastases from colorectal tumours. In: Xie X, Lorca F, Marcon E, eds. Operations Research for Health Care Engineering: Proceedings of the 33rd International Conference on Operational Research Applied to Health Services. Saint-Etienne, France: Publications de l'Universitaire de Saint-Etienne; 2008:493-500.

9. Utley M, Treasure T. Interpreting data from surgical follow-up studies: the role of modeling. J Thorac Oncol. 2010;5(suppl):S200-2.
10. Simms L, Barraclough H, Govindan R. Biostatistics primer: what a clinician ought to know-prognostic and predictive factors. J Thorac Oncol. 2013;8:808-13.

11. Northover J, Houghton J, Lennon T. CEA to detect recurrence of colon cancer. JAMA. 1994;272:31

12. Grunhagen D, Jones RP, Treasure T, Vasilakis C, Poston GJ. The history of adoption of hepatic resection for metastatic colorectal cancer: 1984-95. Crit Rev Oncol Hematol. 2013;86:222-31.

13. Rosen CB, Nagorney DM, Taswell HF, Helgeson SL, Ilstrup DM, Van Heerden JA, Adson MA. Perioperative blood transfusion and determinants of survival after liver resection for metastatic colorectal carcinoma. Ann Surg. 1992;216:493-504.

14. Embun R, Fiorentino F, Treasure T, Rivas JJ, Monlins L, on behalf of Grupo Español de Cirugía Metástasis Pulmonares de Carcinoma Colo-Rectal (GECMP-CCR). Pulmonary metastasectomy in colorectal cancer: a prospective study of demography and clinical characteristics of 543 patients in the Spanish colorectal metastasectomy registry (GECMP-CCR). BMJ Open. 2013;3:e02787.

15. Cooper JD. Randomized clinical trials for new surgical operations: square peg in a round hole? J Thorac Cardiovasc Surg. 2010;140:743-6. 